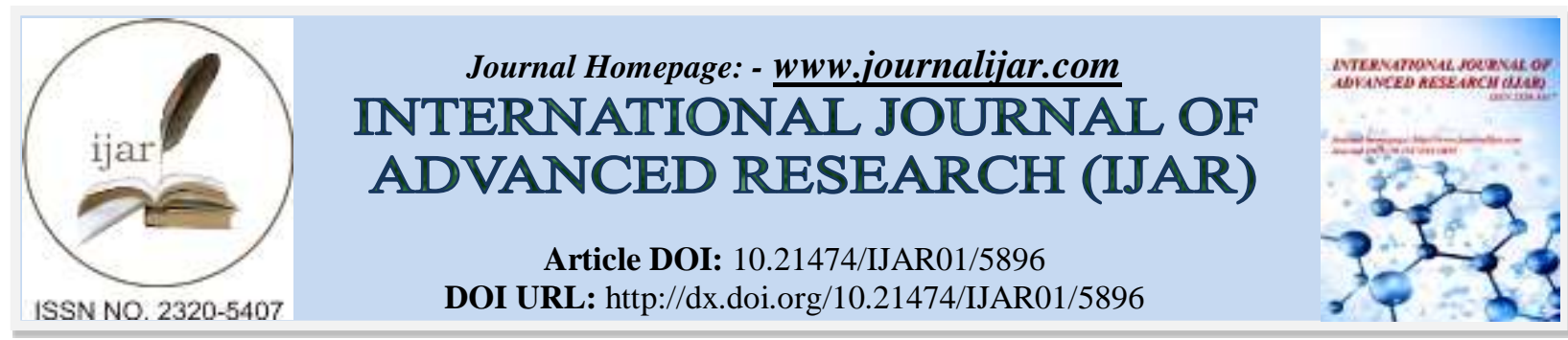

RESEARCH ARTICLE

\title{
RENAL ARTERY EMBOLISM DIAGNOED BY ABDOMINAL CT AND SUCCESSFULLY MANAGED CONSERVATIVELY AFTER THIRTY-SIX HOURS: A CASE REPORT.
}

\author{
"Firas Al Ahmari ${ }^{1}$,Saud Erwi ${ }^{1}$, Ali M Alfageeh ${ }^{1}$, Masoud Awad Alqahtani ${ }^{2}$, Nasser Alhamzi ${ }^{2}$ and Mohammed \\ Saeed Hazzazi ${ }^{2}$. \\ 1. Internal medicine Department. \\ 2. Clinical pathology Department; Armed Forces Hospital Jizan.
}

\section{Manuscript Info}

\section{Manuscript History}

Received: 17 September 2017

Final Accepted: 19 October 2017

Published: November 2017

Key words:-

Renal artery embolism; abdominal CT ;

Conservative management.

\begin{abstract}
Renal artery embolism (RAE) is rarely reported in the literature and is easily missed due to its nonspecific presentations. We report A 67-yearold woman presented with picture suggestive of right renal colic and proved after 36 hours of her onset of symptoms as Renal artery embolism (RAE). Pelvi-abdomenal Contrast-enhanced CT revealed a normal sized nonvasularized right kidney. Patient was managed conservatively with drug therapy in the form of full dose of anticoagulant therapy ( LMW Heparin). We conclude that RAE is not uncommon event and should suspected in old patient with Lion pain. contrast enhanced abdominal CT is fast, and an appropriate choice for the diagnosis of RAE. Moreover anticoagulant therapy is preferred approach with acceptable outcome to avoid unnecessary invasive surgical intervention.
\end{abstract}

Copy Right, IJAR, 2017,. All rights reserved.

\section{Introduction:-}

Renal artery embolism (RAE) is an event that is rarely reported in the literature and is easily misdiagnosed due to its nonspecific presentations [1]

RAE typically associated with atrial fibrillation (AF) [2,3], however other coexisting conditions have been associated with RAE including aneurysmal disease, valvular or ischemic heart disease and advanced malignancy, previous infarction and pulmonary embolism $[2,4]$.

In clinical practice the diagnoses of RAE is often missed on admission. Most patients presented in the sixth decade of life and commonly presented as lion pain, fever, leukocytosis, and hematuria. This presentation can cause diagnostic confusion; initial diagnoses included renal colic, urinary tract infection, pyelonephritis, an acute mesenteric ischemia or biliary tract disease [5]

Different methods of therapy have been described in the last decades including surgical intervention, anticoagulant therapy, systemic and selective thrombolytic therapy. However the Optimal management for RAE still remains controversial [6].

Here, we report A 67-year-old woman who was presented with RAE and was treated successfully by anticoagulant therapy 36 hours after of the event 


\section{Case Report:-}

A 67-year-old woman presented to emergency department with a 12 hours history of right flank sever agonizing pain of sudden onset. The pain not changed in its quality or its intensity since its onset even after the administration of nonsteroidal anti-inflammatory drug (NSAD). She had past history of similar attack with no other systemic symptoms. This patient is a known case atrial fibrillation on regular medication of acetylsalicylic acid and $5 \mathrm{mg}$ warfarin without any prior history of thromboembolic disease.

Physical examination showed, blood pressure $150 / 90 \mathrm{~mm} \mathrm{Hg}$, irregular heart rate of 95 beat / minute, Oxygen saturation $95 \%$ on room-air and body temperature of $37.9^{\circ} \mathrm{C}$. Her abdominal examination was normal except some tenderness in her right flank. Electrocardiogram showed atrial fibrillation with a rate of 95 beat / minute. Laboratory investigation showed the following results: white blood cell (WBC) count $15.4 \times 10^{3}$, platelets 153.000 , hematocrit $30.7 \%$, INR of 1.9 , serum creatinine $127 \mu \mathrm{mol} / \mathrm{L}$ and Urine analysis was unremarkable except for positive occult blood, and 10-20 red blood cells per high powered field. Electrolytes, and liver function tests were normal. The patient received $50 \mathrm{mg}$ Meperidine Hydrochloride in the emergency department and her pain was settled. In view of sudden onset of right flank sever agonizing pain for the second time, absence of signs peritoneal irritation or bowel ischemia and the fact that the patient on regular medication for her atrial fibrillation with INR of 1.9 without any prior history of thromboembolic event, diagnosis of right renal colic with urinary tract infection was made.

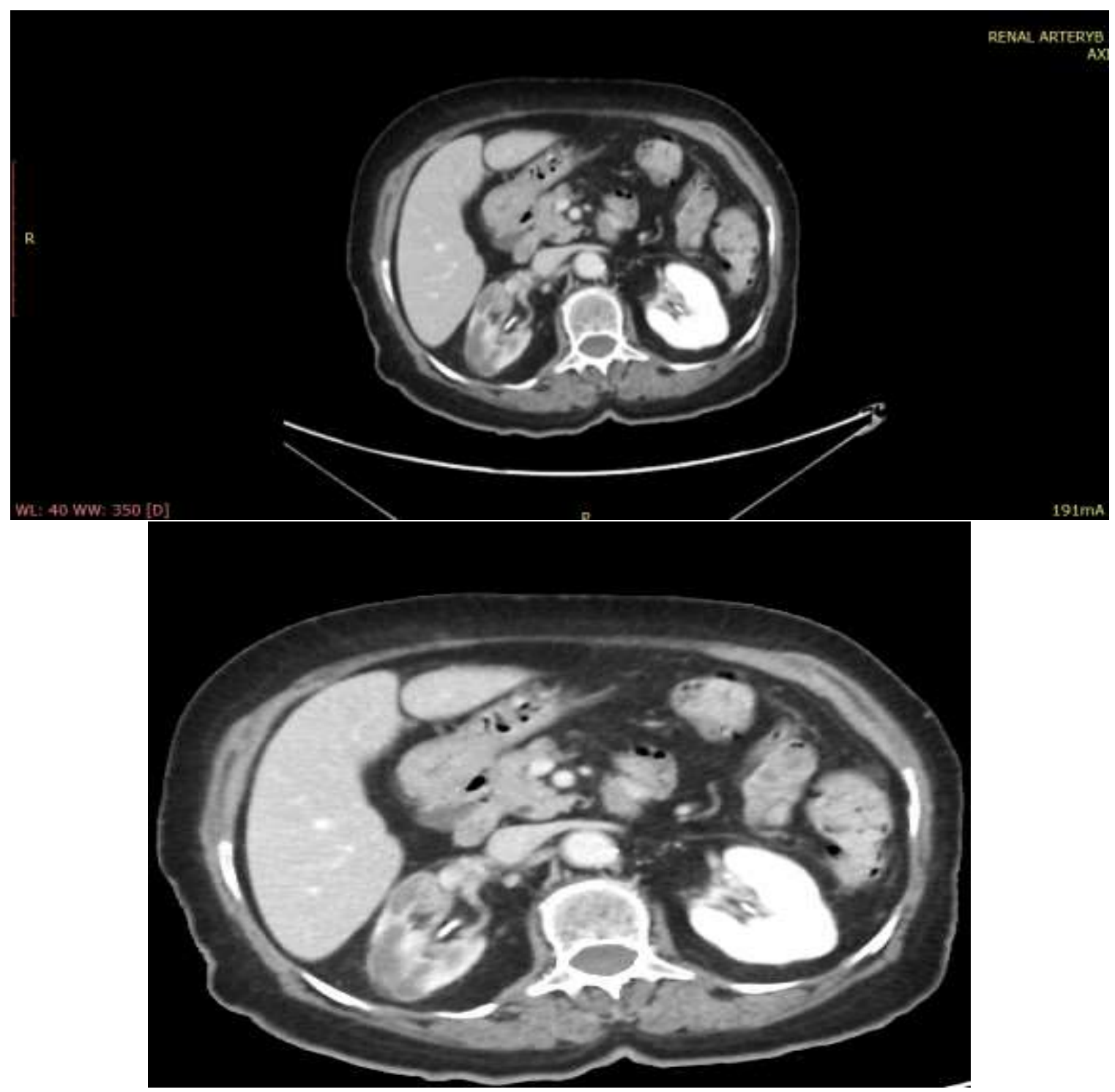

Fig. 1:- Contrast-enhanced abdominal CT scan showing nonvascularized right kidney. 


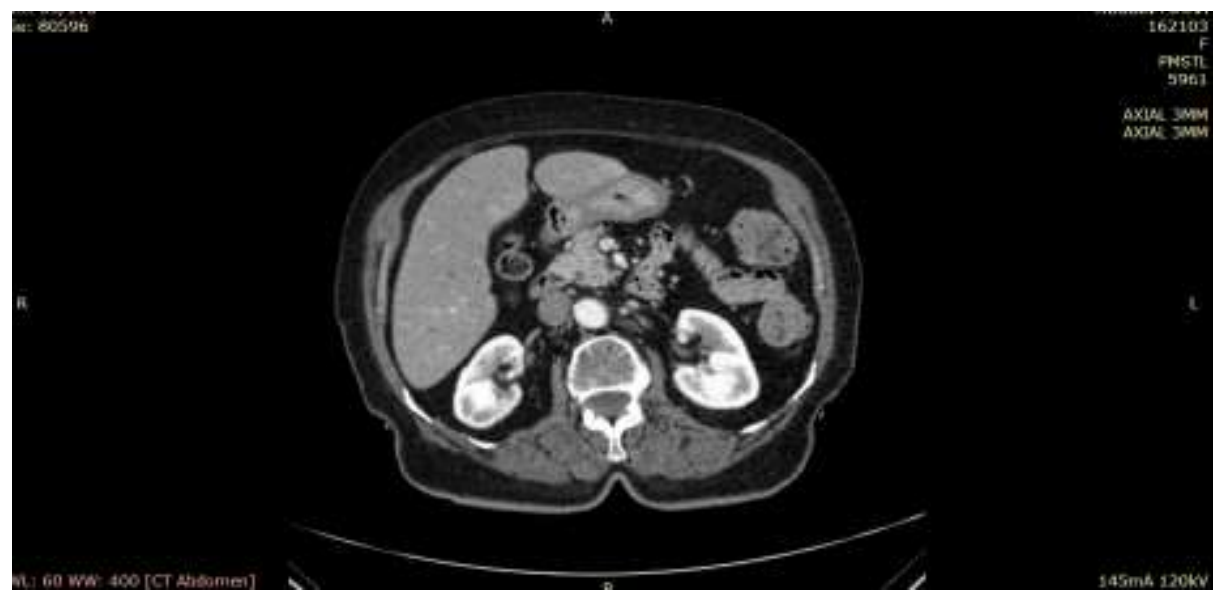

Fig. 2:- Contrast-enhanced abdominal CT scan showing well vascularized right kidney.

Few hours after hospital admission, the pain recurred with the same intensity and the patient recived a second dose of Meperidine Hydrochloride.

Pelvi-abdomenal contrast-enhanced computed tomography (CT) was ordered since the pain recurred after the third Meperidine Hydrochloride dose and revealed a normal sized right kidney with no stones and disappearance of the main stem of the right renal artery, absence of the dye in her right kidney (Figure 1).

A diagnosis of right renal artery embolism was made and the patient received full dose of anticoagulant therapy ( LMW Heparin) for 5 days with continuation of her warfarin therapy. The symptoms settled and the patient was discharged on warfarin therapy with INR 2.4 after seven days of hospital admission without deterioration of her renal function. One month later, follow up Pelvi-abdomenal Contrast-enhanced CT demonstrated complete patency of the renal artery with visualization of the dye allover the right kidney (Fig. 2).

\section{Discussion:-}

Clinical diagnosis of acute RAE is quite difficult and the real incidence of this event is probably underestimated, as post mortem studies have shown that RAE is an underdiagnosed pathology. The reported incidence of renal infarction was $1.4 \%$ in a previous study of 14,411 autopsies, [7].

Since renal insufficiency is seen only in bilateral RAE or in unilateral RAE in whom the contralateral kidney has been removed or non-functioning, the diagnosis of unilateral RAE can be too late and can even go undiagnosed [8]. Major risk factors for RAE include atrial fibrillation, vavular o ischemic heart disease, aortic atheromatosis and previous thromboembolic event [2, 3, 4, 7]. Although our patient is a known case atrial fibrillation which is a well known risk factor for acute RAE, she was diagnosed as a case renal colic. This because she was presented with a second attack similar to renal colic, absence of signs peritoneal irritation and the fact that the patient on regular medication for her atrial fibrillation without past history of thromboembolic event.

To establish the diagnosis of RAE, angiography remains the gold standard investigation but it has the disadvantage that it is an invasive procedure. The diagnosis is confirmed by the demonstration of an occlusion or filling defect in the renal artery on angiography [5,9].

RAE can be also diagnosed by renal ultrasound, abdominal CT scan, a renal isotope scan, or excretory urography. Ultrasound has the advantage of being widely available and inexpensive but the pervious series proved that renal ultrasound seems to be unreliable diagnostic tool for RAE or infarction and should not be used for the diagnosis of this condition due to the low sensitivity $[2,3,5,10]$.

CT scan of the kidney was a more useful investigation in the previous reports to diagnose ARE, where of the 15 tests performed, 12 were positive and 3 negative in one report [5] and in other report 11 patients were diagnosed by contrast-enhanced CT, although 3 patient were initially misdiagnosed [3]. The conclusion was that Contrastenhanced CT scan of the kidney is fast becoming the diagnostic tool of choice for RAE. 
To confirm the diagnose RAE we do not include the use of renal scintigraphy or angiography in our current clinical practice. When our patient did not improved after 36 hours of onset of her symptoms and she is a known case of atrial fibrillation, this raised our suspicion of renal ischemia and Contrast-enhanced Pelvi-abdomenal CT was ordered which confirmed the diagnosis of right renal ischemia (Fig. 1).

Different methods of management have been used in the last decades including, anticoagulant therapy, selective and systemic thrombolytic therapy and surgical intervention [6]. However, there is no agreement to the optimum treatment $[6,11,12]$. Drug therapy are generally preferred, this because the fact that embolectomy or vascular reconstruction is associated with higher mortality rate and no evidence of improvement of renal function [6]. Moreover the use thrombolytic treatment is associated with risks of hemorrhage and poor outcome. An acceptable outcome of renal function is achieved in most of the patients with heparin anticoagulation and subsequently warfarin therapy [5].

Once the diagnosis of our patient was confirmed as right renal artery embolism, anticoagulant therapy with LMW Heparin and warfarin was initiated for 5 day, the patient improved and was discharged on warfarin therapy with INR 2.4 after seven days of hospital admission without deterioration of renal function. One month later, the follow up Contrast-enhanced abdominal CT demonstrated complete patency of the renal artery with visualization of the dye all over the right kidney (Fig. 2).

Different authors [13] suggest that the duration of renal ischemia is an important factor for recovery of renal function, however successful late revascularization and renal recovery can be can be reversed after longer periods of occlusion $[6,8,14,15]$ which may extended up to 5 days ( 8). In reality, this almost certainly reflects the extent of collateral renal circulation [15] .

Although we started the anticoagulant therapy 36 hours of the onset of patient's symptoms, the patient improved with return of her baseline renal function where serum creatinine was $105 \mu \mathrm{mol} / \mathrm{L}$ and the follow up abdominal CT showed well vascularized right kidney (Figure 2.).

Our patient was on warfarin therapy with INR of 1.9 and without past history of embolic event. It is possible to assume that renal artery embolism could be the initial presentation of embolic event in patient with atrial fibrillation, although this condition is rare in the literature.

In a study of 44 patients with atrial fibrillation only 9 patient were treated with warfarin before RAE events, and only 2 of these patients had an INR >1.8 [5]. Considering this study and our patient we suggest that Most of patients with RAE either were undercoagulated as our patient or not treated with warfarin. Moreover we can propose the importance of appropriate warfarin anti coagulation therapy with INR in the therapeutic range (2-3) to prevent thromoembolization in such patients.

\section{Conclusion:-}

Lion or back pain in old patient with a risk factor for thromboembolic disease should raise the suspicion of RAE as it is not uncommon occurrence even without past history of embolic event . Considering our patient and previous reports, contrast enhanced abdominal CT is fast, non invasive and an appropriate choice for the diagnosis of renal artery embolism. Moreover anticoagulant therapy is preferred choice with acceptable outcome of renal function and appropriate warfarin therapy with INR 2-3 can prevent RAE.

\section{Acknowledgment:-}

I take this opportunity to express my deep sense of gratitude to major general dr Sulaiman Al Malik, director of medical services, General Directorate, for his constant encouragement, cordial support throughout the course of my work, valuable information and guidance, which helped me in completing this task through its various stages.

Dr: Firas Al Ahmari

\section{Highlight:-}

Renal artery embolism (RAE) is rarely reported in the literature and is easily missed due to its nonspecific presentations. 
We report A 67-year-old woman presented with Renal artery embolism (RAE),diagnosed after 36 hours of her onset of symptoms by Contrast-enhanced abdomenal C . RAE can be managed conservatively by drug therapy.

We conclude that RAE is not uncommon event. contrast enhanced abdominal CT is fast, and an appropriate choice for the diagnosis of RAE. Moreover anticoagulant therapy is preferred apprach with acceptable outcome to avoid unnecessary invasive surgical intervention.

\section{References:-}

1. Chu PL, Wei YF, Huang JW, Chen SI, Chu TS,Wu KD. Clinical characteristics of patients with segmentalrenal infarction. Nephrology (Carlton) 2006;11(4):336-40.

2. Domanovits H, Paulis M, Nifkardjam M, Meron G, Kurkciyan I, Bankier AA, Laggner AN. Acute renal infarction. Clinical characteristics of 17 patients. Medicine (Baltimore). 1999;78:386-394.

3. Korzets Z, Plotkin E, Bernheim J, Zissin R. The clinical spectrum of acute renal infarction. Isr Med Assoc J. 2002;4:781-784.

4. Lope z VM, Glauser J. A case of renal artery thrombosis with renal infarction. J Emerg Trauma Shock 2010;3(3):302.

5. Hazanov N, Somin M, Attali M, et al. Acute renal embolism: forty-four cases of renal infarction in patients with atrial fibrillation. Medicine (Baltimore) 2004;83(5):292-9.

6. Cheng BC, Ko SF, Chuang FR, Lee CH, Chen JB, Hsu KT. Successful management of acute renal artery thromboembolism by intra-arterial thrombolytic therapy with recombinant tissue plasminogen activator. Ren Fail 2007;25(4):665-70.

7. Hoxie HJ, Coggin CB. Renal infarction: Statistical study of two hundred and five cases and detailed report of an unusual case. Arch Intern Med 1940; 65: 587-594

8. Fort J, Camps J, Ruíz P, et al. Renal artery embolism successfully revascularized by surgery after 5 days' anuria Is it never too late Nephrol Dial Transplant 1996; 11: 1843-5.

9. Piffaretti G, Riva F, Tozzi M, et al. Thrombolysis for acute renal artery thrombosis: report of 4 cases. Vasc Endovasc Surg 2008;42(4):375-9.

10. Lessman RK, Johnson SF, Kaufman JJ. Renal artery embolism: clinical features and long term follow up of 17 cases. Ann Intern Med. 1978; 89:477-482.

11. Gasparini M, Hofmann R, Stoller M. Renal artery embolism: clinical features and therapeutic options J Urol 1992; 147: 567-72.

12. Lacombe M. Surgical versus medical treatment of renal artery embolism J Cardiovas Surg 1977; 18: 281.

13. Hobarth K. Kratzik CH, Schurawitzki H. Diagnosis of renal artery occlusion by duplex sonography and successful lysis therapy. Urol Int 1991; 47: 136-159

14. Gluck G, Croitoru M, Deleanu D, Platon P. Local thrombolytic treatment for renal arterial embolism. Eur Urol 2000;38(3):339-43.

15. Robinson S, Nicholas D, MacLeod A, Duncan J. Acute renal artery embolism: a case report and brief literature review. Ann Vasc Surg 2008;22(1):145-7. 